\title{
A BRUCK-RYSER TYPE NONEXISTENCE THEOREM
}

BY STANLEY E. PAYNE

Communicated by Irving Reiner, March 21, 1968

Let $P$ be a regular, bipartite graph of degree $s+1$ on $2\left(2+s^{+}+s^{2}\right)$ nodes with girth 6 . Equivalently, let $P$ be a $v \times v(3, s, s)$-configuration with $v=2+s+s^{2}$. Then it is known that either $s+1$ or $s-1$ is a perfect square (see [2] and [3] for related definitions and results). Using techniques suggested by [1] we prove the following:

TheOREM. Let $s \equiv 1(\bmod 8)$ and let $p \equiv 3(\bmod 8)$ be a prime dividing the square-free part of $s+1$. Then no $P$ can exist. Also, if $s \equiv 3(\bmod 8)$ and $p \equiv 7(\bmod 8)$ is a prime dividing the square-free part of $s-1$, then no $P$ can exist.

The second statement of the theorem may also be proved by a direct application of the Bruck-Ryser theorem to a $(v, k, \lambda)$-design with $\lambda=2$ which may be obtained by "halving" $P$. Proofs of these and other related results will be given elsewhere.

\section{REFERENCES}

1. J. K. Goldhaber, $A$ note concerning subspaces invariant under an incidence matrix, J. Algebra 7 (1967), 389-393.

2. S. E. Payne, On the nonexistence of a class of configurations which are nearly generalized n-gons (to appear).

3. S. E. Payne and M. F. Tinsley, $O n v_{1} \times v_{2}(n, s, t)$-configurations, J. Combinatorial Theory (to appear).

Miami University 